\title{
Numerical Simulation on Micro-damage Detection In CFRP Composites Based on Nonlinear Ultrasonic Guided Waves
}

\author{
Chang Jiang ${ }^{1, a}$, Weibin $\mathrm{Li}^{1, \mathrm{~b},{ }^{*}, \text { Mingxi Deng }}{ }^{2, \mathrm{c}}$ and Ching-Tai $\mathrm{Ng}^{3, \mathrm{~d}}$ \\ ${ }^{1}$ School of Aerospace Engineering, Xiamen University, China \\ ${ }^{2}$ College of Aerospace Engineering, Chongqing University, China \\ ${ }^{3}$ School of Civil, Environmental \& Mining Engineering, University of Adelaide, Australia \\ ajiangc632@gmail.com, bliweibin@xmu.edu.cn, 'dengmx65@yahoo.com, \\ dalex.ng@adelaide.edu.au
}

Keywords: Nonlinear Guided Waves, Composites, Micro-Damage, Random Defects

\begin{abstract}
Micro-damages such as pores, closed delamination/debonding and fiber/matrix cracks in carbon fiber reinforced plastics (CFRP) are vital factors towards the performance of composite structures, which could collapse if defects are not detected in advance. Nonlinear ultrasonic technologies, especially ones involving guided waves, have drawn increasing attention for their better sensitivity to early damages than linear acoustic ones. The combination of nonlinear acoustics and guided waves technique can promisingly provide considerable accuracy and efficiency for damage assessment and materials characterization. Herein, numerical simulations in terms of finite element method are conducted to investigate the feasibility of micro-damage detection in multi-layered CFRP plates using the second harmonic generation (SHG) of asymmetric Lamb guided wave mode. Contact acoustic nonlinearity (CAN) is introduced into the constitutive model of micro-damages in composites, which leads to the distinct SHG compared with material nonlinearity. The results suggest that the generated second order harmonics due to CAN could be received and adopted for early damage evaluation without matching the phase of the primary waves.
\end{abstract}

\section{Introduction}

Composite materials are gaining increasing interest from researchers for their excellent performances which are superior to metals in aerospace engineering and many other fields. Carbon fiber reinforced plastics (CFRP), as an important type of composites, can provide high-strength, high-durability against corrosion and many other great mechanical properties [1-3]. However, due to its distinct material composition, microstructures and manufacturing process, micro-damages such as micropores, closed delamination/debonding and fiber/matrix crack could occur when subjected to thermal, chemical and mechanical loads [4]. As invisible as those defects could be, long term accumulation of micro damages can lead to sudden structural disaster. Therefore, it is of vital importance to develop advanced non-destructive technologies to detect and evaluate the early damage state of CFRP materials.

Among conventional non-destructive testing techniques, ultrasonic testing methods have been favored over thermal infrared and radiographic ones due to their cost-effective features [5]. Nevertheless, linear acoustic characteristics, such as the appearance of pulse echoes, attenuation and variation of wave velocity, could be too subtle to be applied for early damage assessment. The micro-damages in CFRP plates usually have little influence on the propagation of ultrasonic primary waves. For this problem, promising solutions have been proposed including nonlinear 
ultrasonic techniques, which often involves the second harmonic generation (SHG) resulted from the interaction of material nonlinearity with primary waves [6].

On the other hand, nonlinear guided wave techniques have recently been widely investigated, since point-wise detection with bulk wave transducers can be inefficient, and could not cope with relatively thin plate in which pulse cycles are not sufficient for nonlinearity analysis [7-8]. The combination of ultrasonic nonlinear response with guided waves in micro-damaged material, can potentially enhance the sensitivity and efficiency of the evaluation process. However, one of the main concerns whilst dealing with such applications is the dispersion nature of guided waves. It has been reported that synchronism and non-zero power flux conditions are required for SHG with cumulative effect using a single excitation of primary guided waves [9].

In recent years, the contact acoustic nonlinearity (CAN) caused by local closed defects such as delamination and crack are drawing attentions from researchers [10-11]. This paper mainly aims at exploring the application potential of nonlinear ultrasonic guided waves to assess early damages in CFRP laminated plates. Numerical studies on the CAN caused by randomly distributed micro-defects are conducted. The received ultrasonic response are analyzed in terms of time and frequency domains adopting the primary guided waves of asymmetric Lamb mode. Discussions upon the received nonlinear ultrasonic responses caused by single and multiple micro-damage interfaces are as well provided.

\section{Numerical investigations}

Material, geometry and physics. The composite material adopted in this study are composed of T300/M914 carbon fiber reinforced plastics, of which the thickness is $2 \mathrm{~mm}$. The laminated plates 0are layered as [0/90/0/90]s order. To assure sufficient calculation efficiency and accuracy, the geometry size of the finite element model is $140 \mathrm{~mm} \times 10 \mathrm{~mm} \times 2 \mathrm{~mm}$, as shown in Fig. 1. The left end face of the model is prescribed with Hanning-windowed displacement excitation up to $100 \mathrm{~nm}$. The front, back and right face are set with low reflection boundary condition, which can significantly absorb waves approaching there, and in the meantime enhance the signal-to-noise ratio of primary and harmonic waves.

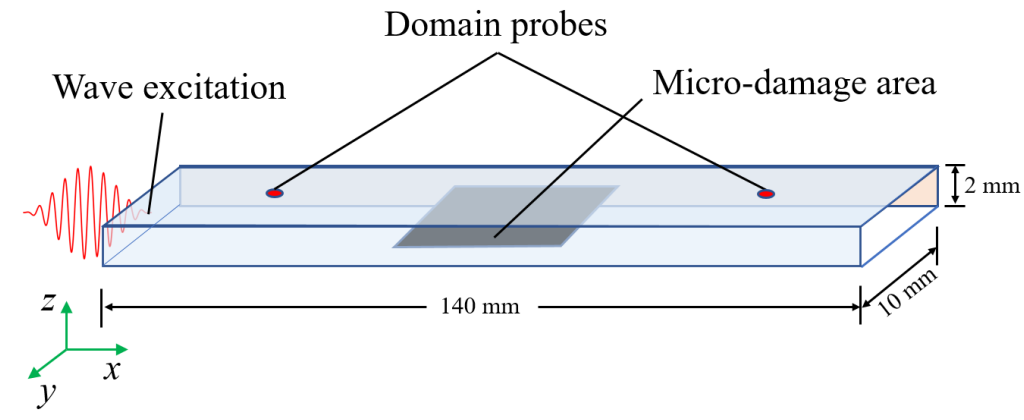

Fig. 1. Illustration of the model geometry and physical setups.

Miro-damage area settings. A number of circular nonlinear interfaces are randomly distributed in the designed damage area, of which the size is $10 \mathrm{~mm} \times 10 \mathrm{~mm}$ and the location is the center of the model in both $\mathrm{x}$ and $\mathrm{z}$ direction. It should be noted that the damage area is a 3D space in the extended simulation as presented in Fig. 2(b). Those interfaces (shown in Fig. 2(a)), of which radii range from $2 \mathrm{~mm}$ to $10 \mathrm{~mm}$, are built and set to be able to decouple the displacements of their two sides with a bilinear stress-strain relation [12]. As a result, they act as local sources of acoustic nonlinearity corresponding to damages such as closed delamination, and can result in 
ultrasonic nonlinear response, which are to be picked up by domain probes deployed in the upper surface of the model.

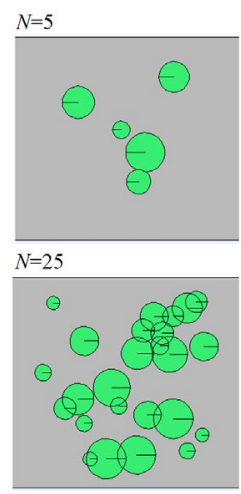

(a)
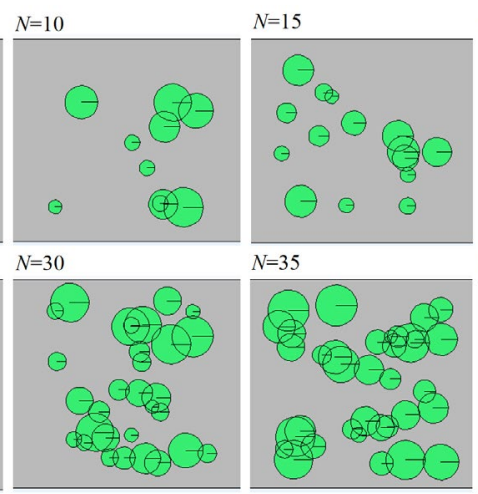

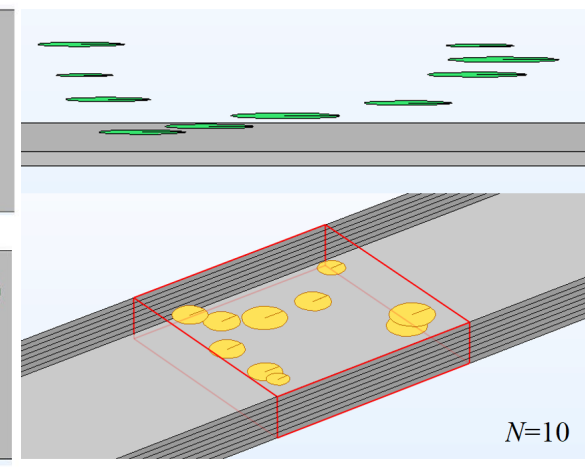

(b)

Fig. 2. Demonstration of the nonlinear mechanical interfaces randomly distributed in the damage area, (a) top view, (b) $3 D$ view.

Guided wave modes. To obtain suitable guided wave modes for signal excitation and receiving, the dispersion curves of such material are plotted as Fig. 3. The frequency of the primary $A 0$ mode guided waves to be excited is chosen as $0.2 \mathrm{MHz}$. Consequently, possible generated second order harmonics through interaction of micro-damages with the primary waves are supposed to be at the frequency of $0.4 \mathrm{MHz}$. As one can see from Fig. 1(a), the phase velocity of primary waves differ from the potential second harmonics. Therefore, the received ultrasonic nonlinear response can include mainly contact acoustic nonlinearity rather than material nonlinearity, referencing [13]. However, according to Fig. 1(b), the potential $A 0$ mode second harmonics share the same group velocity of the primary waves, suggesting that the generated harmonics can be predicted and received by corresponding calculation.
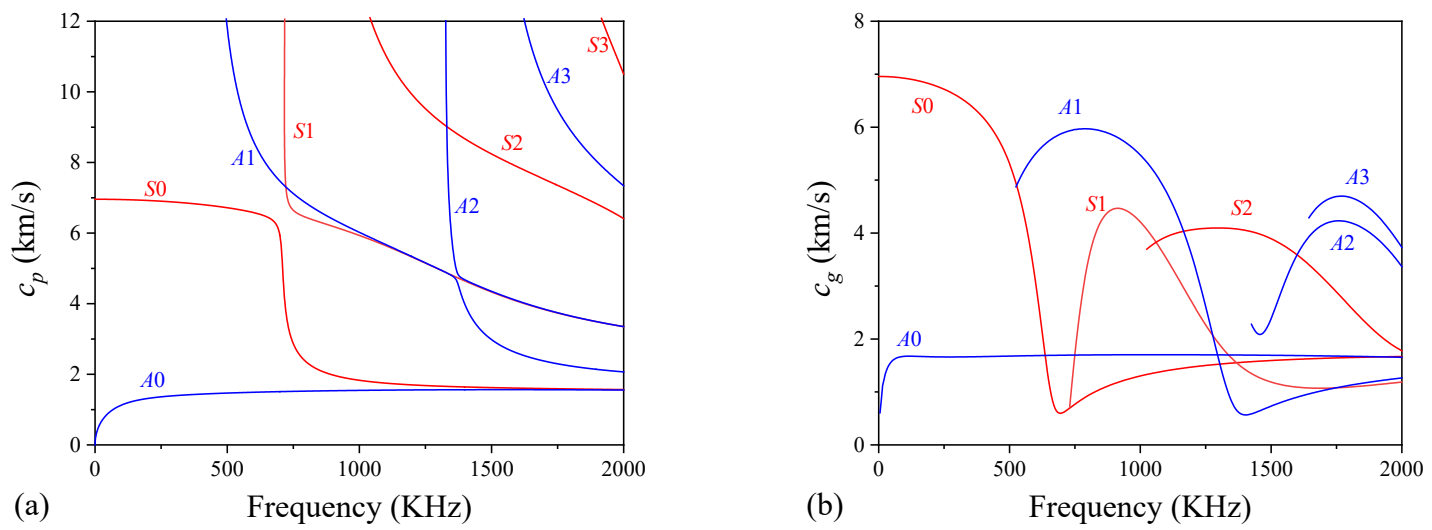

Fig. 3. Dispersion curves of Lamb guided waves in T300/M94 laminated plates, (a) phase velocity, (b) group velocity.

Mesh and solver configurations. The whole model as the wave guide is meshed with free triangle elements, of which the maximum size is defined as $\lambda / 20$. The time step of the transient 
solver is set as $1 / 20 f$ ( $\lambda$ and $f$ is the wavelength and frequency of the primary waves, respectively). The above setups can ensure moderate accuracy of the numerical results.

\section{Results \& Discussions}

Verification of contact acoustic nonlinearity. Firstly, a single nonlinear mechanical interface of varying depth and size is introduced into the model for investigation of CAN. As shown in Fig. 4(a), the group velocity of the primary waves is calculated as about $1600 \mathrm{~m} / \mathrm{s}$, which is in accordance with that of $A 0$ mode waves at the frequency of $0.2 \mathrm{MHz}$. With FFT applied to the 10 -cycle transmission waves received at $\mathrm{x}=0.105 \mathrm{~m}$, the frequency spectrum is obtained as shown in Fig. 4(b). It is noted that there is no second harmonics generated in a model without nonlinear interfaces set in $(R=0 \mathrm{~mm})$, and the magnitude of the second harmonics increase with the radius of the damage interface (the depth is fixed as $0.2 \mathrm{~mm}$ ) as presented in Fig 4(b) and Fig. 5(a). Besides, the Fig. 5(b) shows a trend that the closer the nonlinear interface is to the center of the model along thickness direction, the greater the magnitude of the generated second harmonic waves is. Such result could be related to the wave structure of the primary wave mode.
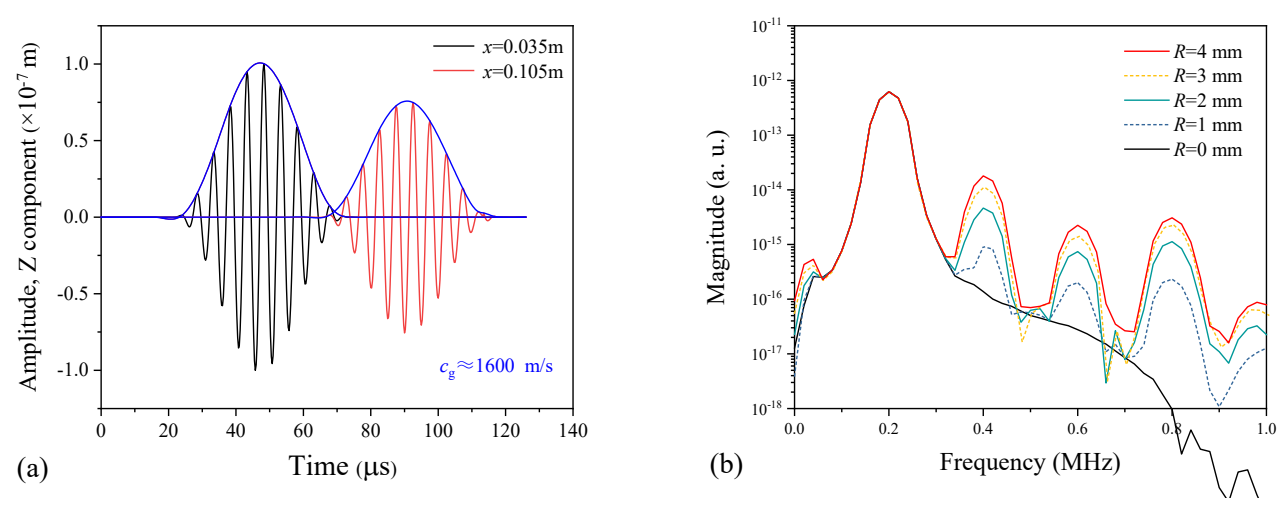

Fig. 4. Time domain (a) and frequency domain (b) of the received guided wave signals from two probes.
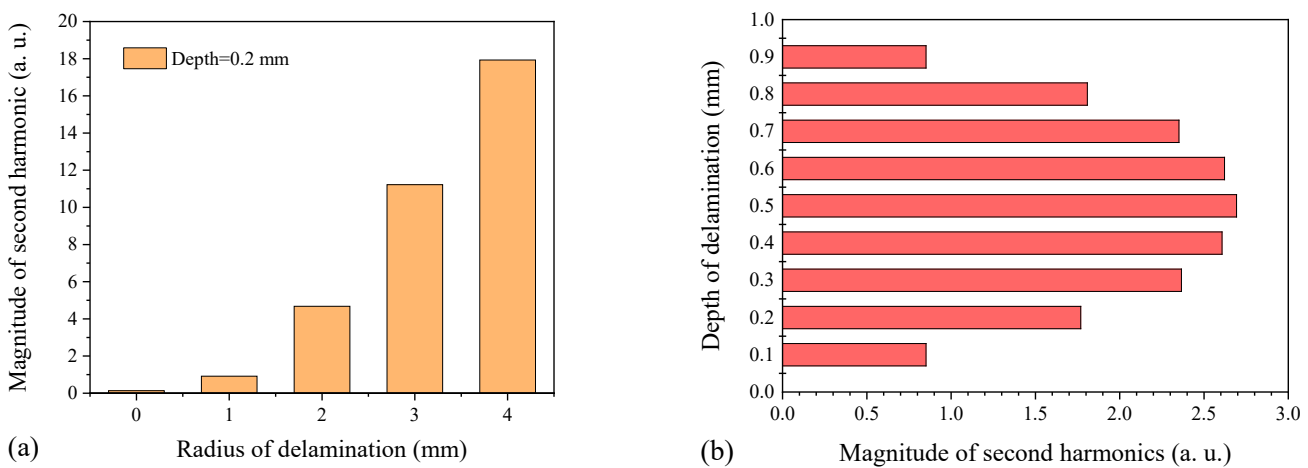

Fig. 5. Parametric studies of the relation between the magnitude of second harmonics and a single closed delamination modeled by a nonlinear mechanical interface through change of the (a) radius, (b) burial depth. 
Furthermore, two other investigations are implemented while a number of the nonlinear interfaces are randomly distributed in the designed micro-damage plane field and the three dimensional space, respectively. Fig. 6 and Fig. 7 show the numerical simulation results. As seen from Fig. 6, the magnitude of the newly generated harmonics at the frequency of $0.4 \mathrm{MHz}$ increase with the number $(N)$ of the artificial damage interfaces which are distributed in the designed plane field located in the middle depth of the laminate. On the other hand, the almost same result applies to the case in which the interfaces are randomly distributed in the 3D damge area (see Fig. 2(b)), as seen in Fig. 7. It is noted that when $N=5$ the magnitude of the quatratic nonlinearity in the receieved trasansmission waves is a bit greater than that when $N=10$, as shown in Fig. 7(b). This could mainly be due to that the different depths of the interfaces influence more on the second harmoic generation than the number of defects when there is obviously less of them buried in the model. However, if a large number of micro-defects such as pores and micro-delaminations occur in a relatively concentrated area, the total nonlinear ultrasonic response measured by SHG will be satisfactory index of the number of the damages regardless of their burial depth.
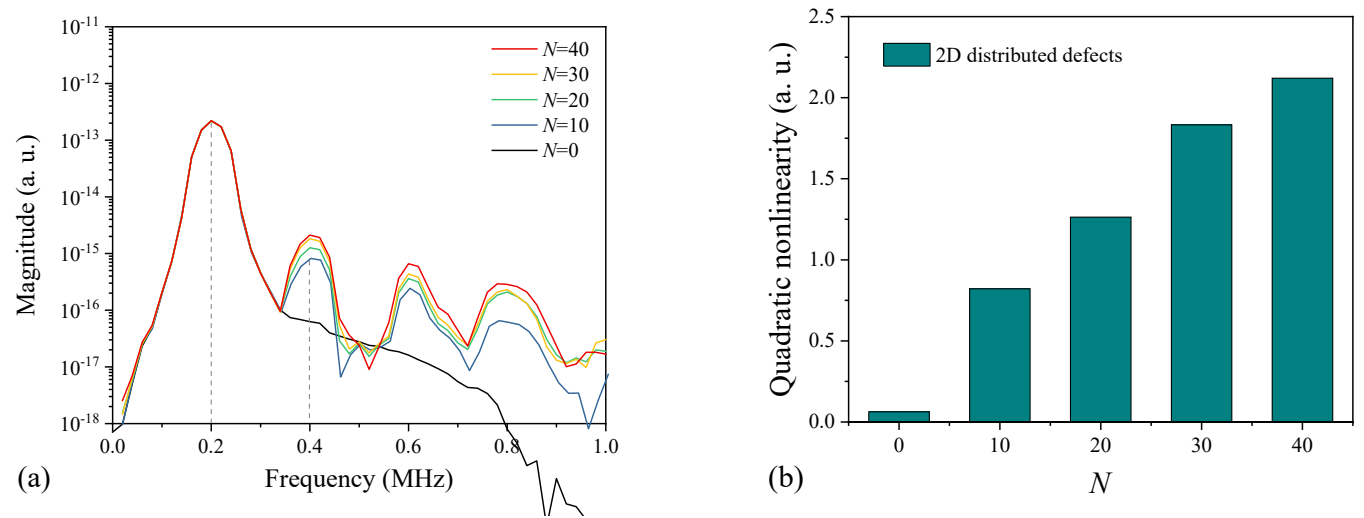

Fig. 6. Frequency domain (a) and the magnitude comparison of the quadratic nonlinearity (b) of the received transmission waves while defects are laid out in the plane damage area.
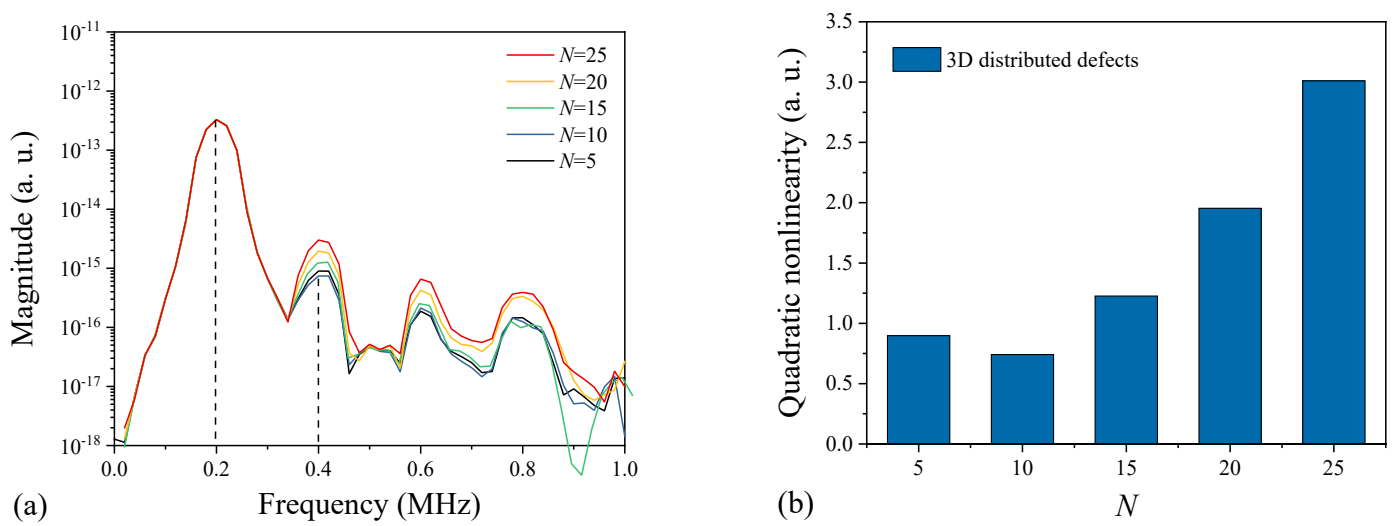

Fig. 7. Frequency domain (a) and the magnitude comparison of the quadratic nonlinearity (b) of the received transmission waves while defects are laid out in the $3 D$ damage area.

\section{Conclusion}

This paper mainly focuses on the feasibility study of micro-damage detection in carbon fiber reinforced plastics using second harmonics generation induced by contact acoustic nonlinearity. 
Nonlinear stress-strain relationship is introduced in the model of damage interface, and ultrasonic nonlinear responses involving Lamb guided waves are analyzed in terms of time domain and frequency domain. Results show that the contact acoustic nonlinearity in CFRP composites can be strong sources of nonlinear acoustic response, which can be detected through the measure of higher harmonics without being limited by the phase matching condition.

\section{References}

[1] Zhao JL, Chillara VK, Ren BY, et al. Second harmonic generation in composites: Theoretical and numerical analyses. Journal of Applied Physics. 2016. https://doi.org/10.1063/1.4941390

[2] Kim EH, Rim MS, Lee I, et al. Composite damage model based on continuum damage mechanics and low velocity impact analysis of composite plates. Composite Structures. 2013. https://doi.org/10.1016/j.compstruct.2012.07.002

[3] Zhao GQ, Wang B, Wang T, et al. Detection and monitoring of delamination in composite laminates using ultrasonic guided wave. Composite Structures. 2019. https://doi.org/10.1016/j.compstruct.2019.111161

[4] Wang B, Zhong SC, Lee, TL, et al. Non-destructive testing and evaluation of composite material/structures: A state-of-the-art review. Advances in Mechanical Engineering. 2017. https://doi.org/10.1177/1687814020913761

[5] Marcantonio V, Monarca D, Colantoni A, et al. Ultrasonic waves for materials evaluation in fatigue, thermal and corrosion damage: A review. Mechanical Systems and Signal Processing. 2019. https://doi.org/10.1016/j.ymssp.2018.10.012

[6] Scott K, Kim JY, Jacobs L. Signal processing methods for second harmonic generation in thin specimens. NDT and E International. 2018. https://doi.org/10.1016/j.ndteint.2018.02.001

[7] Zhu WJ, Xiang YX, Liu CJ, et al. A feasibility study on fatigue damage evaluation using nonlinear Lamb waves with group-velocity mismatching. Ultrasonics. 2017.

https://doi.org/10.1016/j.ultras.2018.06.002

[8] Li WB, Cho Y. Combination of nonlienar ultrasonic and guided wave tomography for imaging the micro-defects. Ultrasonics. 2016. https://doi.org/10.1016/j.ultras.2015.10.016

[9] Hasanian M, Lissenden CJ. Second order harmonic guided wave mutual interactions in plate: Vector analysis, numerical simulation, and experimental results. Journal of Applied Physics. 2017. https://doi.org/10.1063/1.4993924

[10] Solodov IY, Krohn N, Busse G. CAN: an example of nonclassical acoustic nonlinearity in solids. Ultrasonics. 2002, 40:621-625. https://doi.org/10.1016/S0041-624X(02)00186-5

[11] Aleshin V, Delrue S, Trifonov A, et al. Two dimensional modeling of elastic wave propagation in solids containing cracks with rough surfaces and friction - Part I: Theoretical background. Ultrasonics. 2018, 82:11-18. https://doi.org/10.1016/j.ultras.2017.07.002

[12] Broda D, Staszewski WJ, Martowicz A, et al. Modeling of nonlinear crack-wave interactions for damage detection based on ultrasound - A review. Journal of Sound and Vibration. 2014, 333:1097-1118. https://doi.org/10.1016/j.jsv.2013.09.033

[13] Li WB, Deng MX, Hu N, et al. Theoretical analysis and experimental observation of frequency mixing response of ultrasonic Lamb waves. Journal of Applied Physics. 2018, 124:044901. https://doi.org/10.1063/1.5028536 